\title{
Leiomyosarcoma of Rectum Mimicking Primary Ovarian Tumour
}

\author{
Junu Devi \\ Department of Pathology.Assam Medical College.Dibrugarh.Assam,India
}

\begin{abstract}
Leiomyosarcoma of rectum is a very rare mesenchymal tumour with an incidence of approximately $0.1 \%$ of all colorectal malignancies. Because of its rarity, its diagnosis, treatment and pathology often present challenges to the clinicians. The characteristics of this tumour, such as its anatomical location, heterogenous solid features on imaging and non specific lower gastrointestinal tract symptoms can be confused with those of a primary ovarian tumour. Further, a synchronously growing primary ovarian tumour may add more confusion to the diagnosis. We have reported this case of rectal leiomyosarcoma with synchronously occuring primary ovarian tumour in an elderly female presenting with a large lower abdominal mass; where clinical and radiological findings are more suggestive of a primary malignant ovarian tumour with rectal metastasis. In such situation the possibility of other primary tumor of GIT specifically leiomyosarcoma should be looked for.Here Postoperative histopathological examination and immunohistochemistry gives confirmatory diagnosis.
\end{abstract}

Keywords: Ovarian Tumor, Leiomyosarcoma, Rectum, Histopathology, Immunohistochemistry

\section{Introduction}

Rectal leiomyosarcoma is a rare malignant mesenchymal tumour accounting for less than $0.1 \%$ of all malignancies of the colon and rectum. ${ }^{[1]}$ Because of its rarity, its diagnosis, treatment and pathology often present challanges to the clinician. These tumours typically occur in the fifth and sixth decades of life and show a male predominance. ${ }^{[2]}$ Most of the time accurate diagnosis of rectal leiomyosarcoma is difficult because their clinical presentation can mimic that of primary ovarian carcinoma. ${ }^{[3]}$ The characteristics of this tumour such as its anatomical location, heterogenous solid features on imaging and non specific lower GIT symptoms can be confused with those of a Primary Ovarian tumour. Very few cases of rectal leiomyosarcoma have been reported. Here we report a case where there is synchronous growth of a primary Ovarian tumour with a rectal leiomyosarcoma.

\section{Case Report}

A sixty three year old post menopausal woman was admitted in the gynaecology department with the complaints of lower abdominal swelling for past 6 months. In addition she had experienced lower back pain, irregular bowel habit and three episodes of bleeding per rectum. General examination, other system based examination and findings of routine haematological, biochemical and chest radiography were normal .Contrast enhanced CT revealed large abdominopelvic solid to cystic mass having multiple loculations and arising from left adnexa. In addition a large polypoidal mass lesion was noted in the recto sigmoid area which was thought to be an extension of the adnexal mass. Pre-operatively biopsy was obtained from rectal growth and it was reported as malignant spindle cell tumour. Estimation of CA-125 level was within normal limit.

The patient underwent total hysterectomy with removal of the adnexal mass and rectosigmoidal growth. Grossly, a specimen of total hysterectomy with ovarian tumour and part of recto-sigmoid region with a polypoidal intraluminal growth was received. Rectal growth measured 10x $7 \times 5 \mathrm{~cm}^{3}$ (Fig 1). Histologically, rectal growth was composed of fascicles of pleomorphic large spindle cells with moderate to abundant eosinophilic cytoplasm, oval to elongated atypical nuclei, frequently blunt ended, along with multiple mitotic figures (Fig 2 ). Mitotic count was $>10 / 10$ high power fields. Focal areas of necrosis were also noted. Immunohistochemistry (IHC) showed positivity for smooth muscle actin(SMA) (Fig 3a) and negative for CD117 (cKIT)( Fig 3b). Based on the histopathological and immunohistochemical examination a diagnosis of leiomyosarcoma of rectum was made. No evidence of regional or distant metastasis was found. Large adnexal solid cystic mass was diagnosed as ovarian mucinous cystadenoma (Fig 4). Sections from cervix, endomyometrium, other sided ovary showed no specific abnormalities. Both adnexa were free from metastasis. Post operative period was uneventful and later on she was referred to oncology department for further treatment. Now the patient is on regular follow up and is doing well. 


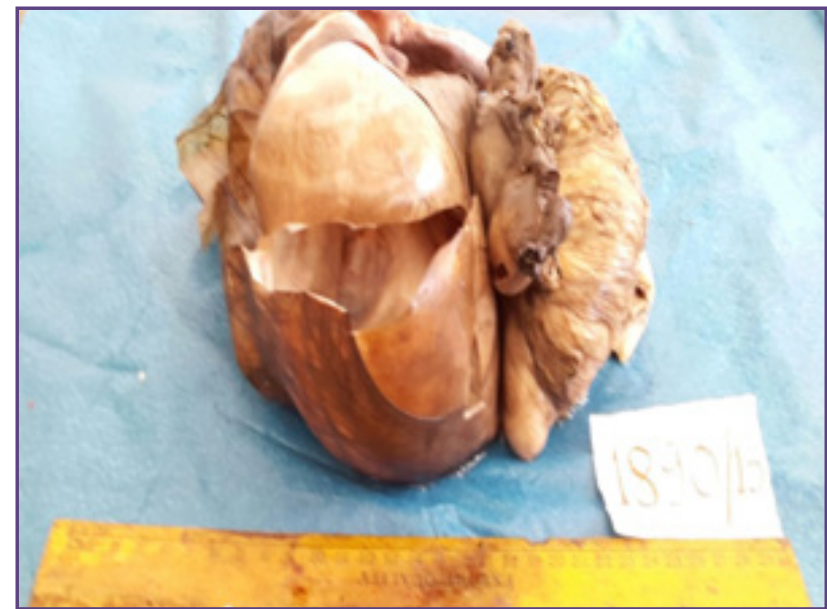

Fig. 1: Specimen of enmass ovarian tumour with uterus and part of recto-sigmoid region showing rectal growth.

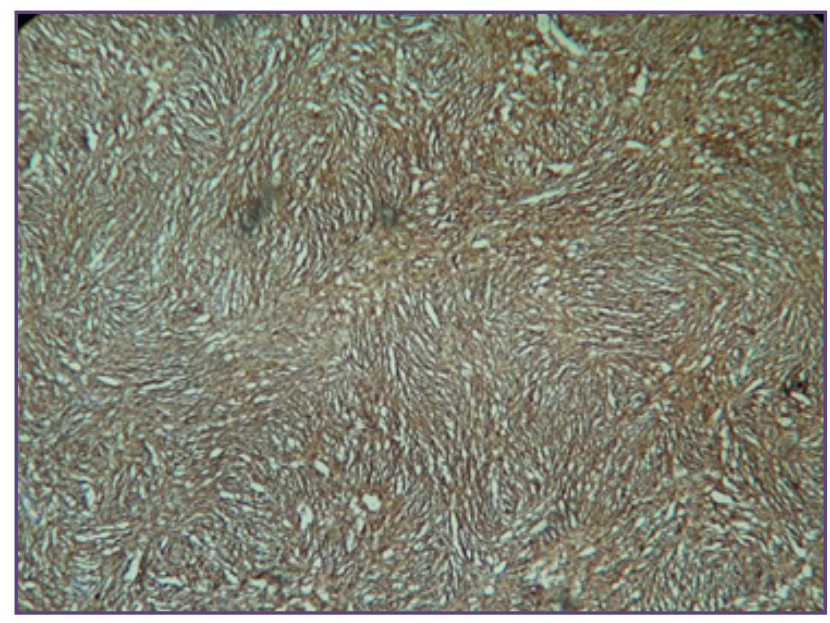

Fig. 3(a): Photomicrograph of leiomyosarcoma showing diffuse strong positivity for smooth muscle actin.

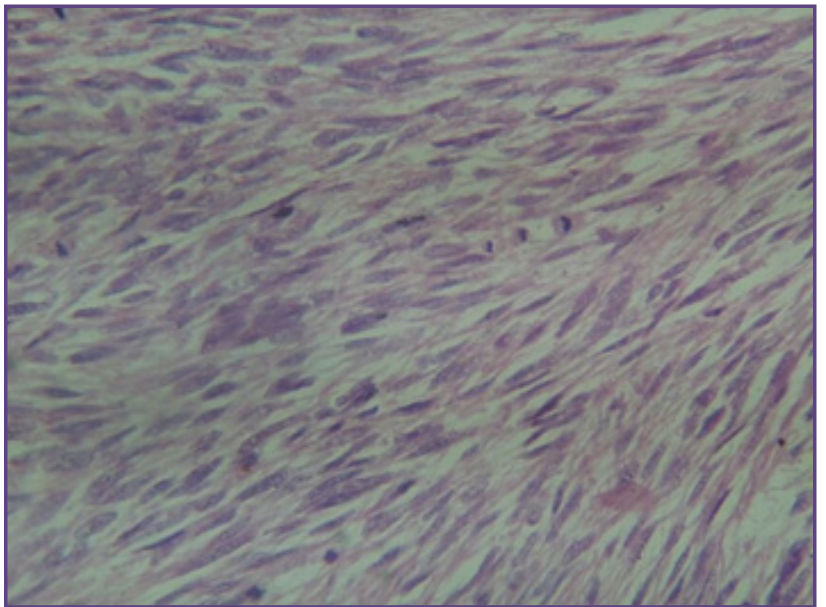

Fig. 2:Photomicrograph of leiomyosarcoma showing fascicles of tumour cells with mitotic figures.(H\&E.10X40).

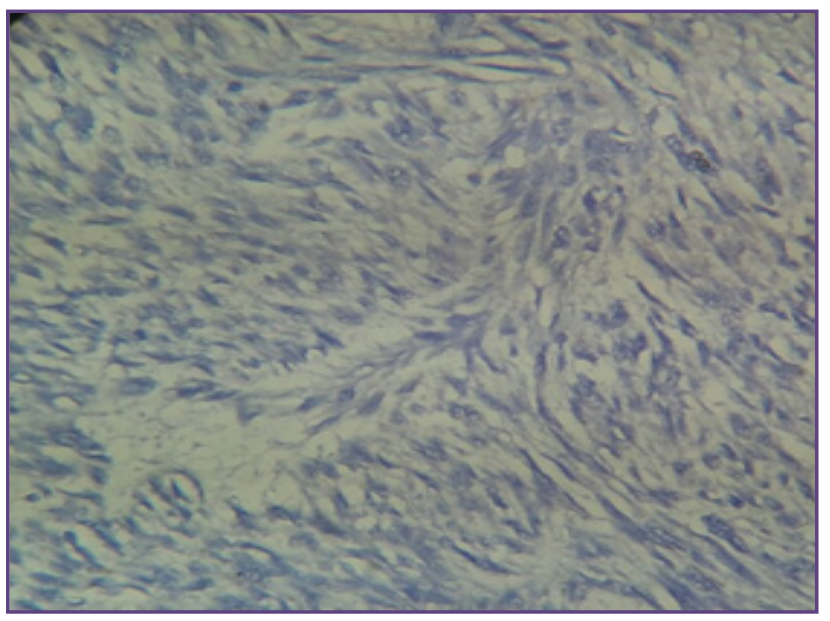

Fig. 3 (b): Photomicrograph of leiomyosarcoma showing IHC negativity for CD117(cKIT).

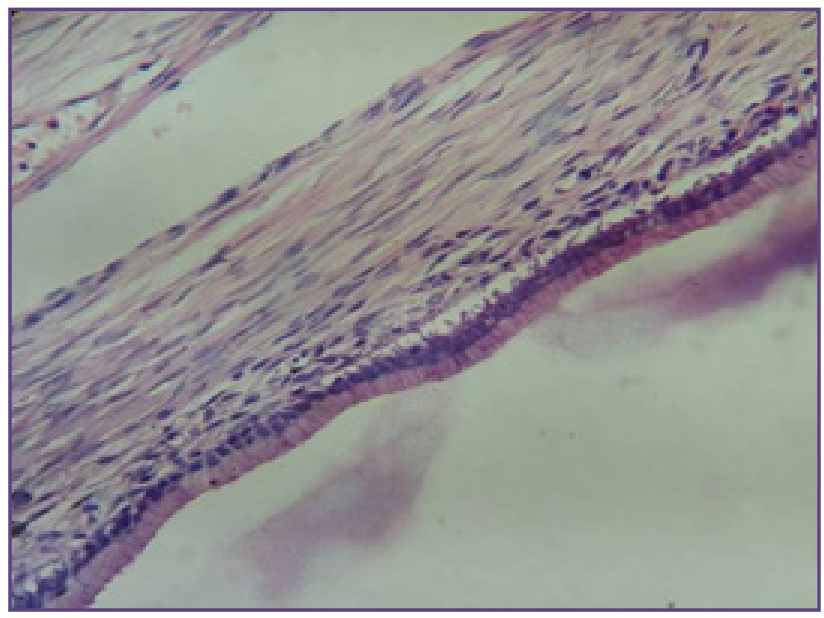

Fig. 4: Photomicrograph of Mucinous cystadenoma of ovary(H\&E 10X40). 


\section{Discussion}

Leiomyosarcomas account for $10-20 \%$ of all soft tissue sarcomas. They arise most frequently from the uterus, gastrointestinal tract and retroperitoneal region. ${ }^{[4]}$ However those arising from the rectum are very uncommon and account for less than $0.1 \%$ of all colorectal malignancies. ${ }^{[1]}$ Yung -taek et al 2013 ${ }^{[3]}$ reported one case of rectal leiomyosarcoma mimicking primary ovarian carcinoma where preoperative diagnosis was primary ovarian carcinoma but postoperative pathological diagnosis came out as leiomyosarcoma of rectum. N. Sahil et al ${ }^{[5]}$ 2016 reported a case of rectal leiomyosarcoma, where preoperative diagnosis was nonspecific, postoperative pathological diagnosis was confirmatory. Evan $\mathrm{s}^{[6]}$ reviewed 56 cases of smooth muscle neoplasm of gastrointestinal tract followed for ten years, only 4 of them were located in the rectum. Randleman et al ${ }^{[7]}$ reported a series of 22 cases of rectoanal leiomyosarcoma seen over 35 years and Walsh et $\mathrm{al}^{[8]}$ reported a series of 48 anorectal leiomyosarcoma in 31 years.

Rectal leiomyosarcoma is associated with rectal bleeding and pain ${ }^{[9]}$. Radiological differentiation between leiomyosarcoma and leiomyoma is difficult. Overlapping of many radiological findings are also commonly encountered in patients with associated ovarian tumour. Hence preoperative radiological differentiation between ovarian tumour and rectal leiomyosarcoma is difficult. ${ }^{[10]}$ Therefore final diagnosis needs to be confirmed by pathological examination. But superficial biopsy specimen may not be representative of the entire tumor and leiomyosarcomas may be misdiagnosed as leiomyomas. Some studies suggest the preoperative histological diagnosis of rectal tumor is adequate in only $29 \%$ of cases. ${ }^{[11]}$ In our case preoperative histological diagnosis was given as malignant spindle cell tumor. Therefore final diagnosis needs to be confirmed by postoperative histopathological examination and immunohistochemical(IHC) analysis. On gross examination leiomyosarcomas are usually large and of a firm consistency, adherent to surrounding tissues. Foci of necrosis, haemorrahage and cystic degeneration may be seen. ${ }^{[12]}$ On histologic examination typical leiomyosarcoma is characterized by sheets of densely packed spindle cells; cells are elongated with moderate to abundant eosinophilic cytoplasm. Centrally placed nucleus is usually blunt shaped or cigar shaped. In some cells perinuclear vacuoles are seen. In poorly differentiated tumor nucleus is more pleomorphic. Mitosis, necrosis, haemorrhage are more common in high grade tumors. On immunohistochemical examination these tumors express smooth muscle cell markers: sooth muscle actin (SMA),smooth muscle myosin heavy chain (SMMHC), h- caldesmon and recently transgelin . ${ }^{[13]}$
Leiomyosarcomas can be differentiated from leiomyoma, schwannoma, and gastrointestinal stromal tumor(GIST) on the basis of histopathology and immunohistochemistry. Among light microscopic findings the presence of mitosis is the hallmark of malignancy ( 5 or more mitosis per 10 high power fields). ${ }^{[14]}$ In the past, most of the Gastrointestinal Stromal tumours (GIST) were diagnosed as Leiomyosarcomas. Immunohistochemistry (IHC) plays a very important role in differentiating it from Gastrointestinal stromal tumor(GIST). Diffuse strong positivity of this tumour for SMA and negativity for CD117 differentiates it from GIST. ${ }^{[15]}$

The spread of rectal leiomyosarcoma is mainly local or haematogenous. Lymphatic metastasis also reported in some tumors. ${ }^{[10]}$ In our case no metastasis was found in resected regional lymphnodes. Prognosis of leiomyosarcoma is poor. Tumor size and degree of differentiation are known to be the most significant prognostic factor. Treatment of rectal LMS is controversial .Radical surgery, such as anterior resection or abdominoperineal resection, is preferred to wide local excision. Radical surgery is associated with a lower recurrence rate. Kalifa et al ${ }^{[2]}$ reviewed 135 cases of rectal leiomyosarcoma, found local recurrence rate of $67.5 \%$ with local excision compared to only $19.5 \%$ with abdominoperineal resection but there were no differences in survival rates. ${ }^{[2,14]}$ Rectal leiomyosarcoma are chemoresistant and radio-resistant. Five year survival rate is $20 \%$. [16]

\section{Conclusion}

This case report points out the diagnostic problem of rectal leiomyosarcoma, particularly when there is a synchronous ovarian tumour. At the same time it also depicts the role of histopathological examination and immunohistochemical analysis in proper diagnosis. Leiomyosarcoma has poor prognosis so early diagnosis and prompt surgical intervention is advocated.

\section{References:}

1. Minsky BD, Cohen AM, Hajdu SI: Conservative management of anal leiomyosarcoma.Cancer 1991,68:16401643.

2. Kahlifa AA, Bong WL, Rao VK, Williams MJ: Leiomyosarcoma of the rectum.Report of a case and review of the literature. Dis Colon Rectum 1986,29:427-432

3. Yung-Tae Ouh, Jin Hwa Hong, Kyung- Jin Min, KyeongA So and Jae Kwan Lee. Leiomyosarcoma of the rectum mimicking primary ovarian carcinoma: a case report .Journal of Ovarian Research 2013, 6:27 http://www.ovarianresearch. com/content $/ 6 / 1 / 27$

4. Talikoti MA, Deo SS, Shukla NK, Kallianpur AA, Gupta M: A rare case of giant leiomyosarcoma in a filarial scrotum: A case report.World J Surg Oncol 2011,9:20. 
5. N. Sahil et al : Unusual evolution of leiomyosarcoma of the rectum: a case report and review of the literature. Journal of Medical case Report 2016,10:249

6. Evans HL . Smooth muscle tumor of the gastrointestinal tract. A study of 56 cases followed for a minimum of 10 years . Cancer 1985:56:2242-50.

7. Randleman CD Jr, Wolff BG, Dozois RR, et al. Leiomyosarcoma of the Rectum and anus. A series of 22 cases. Int J Colorectal Dis 1989;4:91-6.

8. Walsh TH, Mann CV. Soomth muscle neoplasm of the rectum and anal canal. Br J Surgery 1984;71:597-9.

9. Friesen R, Moyana TN, Murray RB, et al. Colorectal leiomyosarcoma: a Patholobiologic study with long term follow-up. Can J Surg 1992:35:505-8.

10. Lee SH, Ha HK, Byun JY, Kim AY, Cho KS, Lee YR, Park HW, Kim PN, Lee MG, Auh YH: Radiological features of leiomyomatous tumours of the colon and rectum.J Comput Assist Tomogr 2000,24:407-412.
11. Chiara O, Canini T, Segala M, Tiberio GA, Gulini SM, Tiberio G. Smooth muscle - cell tumor of the gastroenteric tract. A review of cases. Minerva Chir. 1997;52(10):1147-55.

12. Weiss SW, Goldblum JR . Enzinger and Weiss's soft tissue tumors. 5th ed. St Louis:Mosby; 2008.p 546-55.

13. Rbin YM, Pnel N, Perot G, et al. Transgelin is a noval marker of smooth muscle differentiation that improves diagnostic accuracy of leiomyosarcoma: a comparative immunohistochemical reappraisal of myogenic marker in900 soft tissue tumors. Mod Pathol. 2013;26(4):502-10.

14. Nemer FD, Stoeckinger JM, Evan OT. Smooth muscle rectal tumors: A therapeutic dilemma. Dis Colon Rectum.1977;20:405-13.

15. Christopher D.M. Fletcher. Diagnostic Histopathology of tumours Fourth edition Elsevier Philadelphia. 2013

16. Eitan N, Auslander L, Cohen Y. Leiomyosarcoma of the rectum : report of three cases. Dis Colon, Rectum 1978;21:444-6.

*Corresponding author:

Dr Junu Devi, C-3 Kanika Apartment.Noonmati.Lastgate.Guwahati.Assam- India.PIN 781020

Phone: +91-0361 2656521

Email: drjdevipath@gmail.com

Financial or other Competing Interests: None. 